\title{
Calculation of the $0^{+}$and $2^{+}$Giant Resonances in $\mathrm{Ni}^{58}$
}

\author{
Doris H. Jakubaßa \\ Physik-Department, Technische Universität München, Garching
}

Received February 21, 1974

Abstract. Estimates of the excitation energy, width and sum rule fraction of the collective monopole and quadrupole vibrations indicate that the peak in the $62 A^{-1 / 3} \mathrm{MeV}$ region is a superposition of the $0^{+}$and $2^{+}$modes.

Experiments on the inelastic scattering of electrons and protons on various target nuclei [1] have shown a giant resonance with energy around $62 A^{-1 / 3} \mathrm{MeV}$. Angular distributions were consistent with either a $0^{+}$or a $2^{+}$assignment. The form factors of the two multipolarities were found to differ from each other when the beam was polarized. Therefore new experiments have been performed on $\mathrm{Ni}^{58}$ using a polarized proton beam [2].

In order to identify this giant resonance we present calculations of the collective $0^{+}$and $2^{+}$vibrations in $\mathrm{Ni}^{58}$ and compare them with experiment.

The excitations of a nucleus can be described within the shell model taking into account one particle-one hole excitations. We restricted our configuration space to the levels shown in Table 2. The single particle energies and corresponding wave functions are taken as eigenvalues and eigenvectors of a Woods Saxon potential diagonalized in the space of the harmonic oscillator functions [3].

The potential parameters are chosen to fit the energies of the shells near the Fermi level to the experimental

Table 1. Parameters of the Woods Saxon potential

\begin{tabular}{llllllll}
\hline & $\begin{array}{l}V_{0} \\
{[\mathrm{MeV}]}\end{array}$ & $\begin{array}{l}R_{0} \\
{[\mathrm{fm}]}\end{array}$ & $\begin{array}{l}a_{0} \\
{[\mathrm{fm}]}\end{array}$ & $\lambda$ & $\begin{array}{l}R_{\mathrm{ls}} \\
{[\mathrm{fm}]}\end{array}$ & $\begin{array}{l}a_{\mathrm{ss}} \\
{[\mathrm{fm}]}\end{array}$ & $\begin{array}{l}R_{c} \\
{[\mathrm{fm}]}\end{array}$ \\
\hline Protons & -48.56 & 5.27 & 0.660 & 24.5 & 4.80 & 0.5 & 4.9 \\
Neutrons & -49.16 & 4.94 & 0.535 & 28.1 & 4.84 & 0.5 & 0.0 \\
\hline
\end{tabular}

excitation energies of the neighbouring nuclei. The parameters defined as in Ref. 3 are given in Table 1. Table 2 shows the single particle energies.

The energies of the collective modes were obtained with the RPA method. To simplify the calculations we reduced the problem to that of a closed shell nucleus by interchanging the neutron $2 p_{3 / 2}$ and $2 p_{1 / 2}$ level. A zero range residual interaction was used

$$
\begin{aligned}
& V(1,2)=V_{0} \delta\left(\mathbf{r}_{1}-\mathbf{r}_{2}\right) \\
& \cdot\left(F_{0}+F_{0}^{\prime} \tau_{1} \tau_{2}+G_{0} \sigma_{1} \sigma_{2}+G_{0}^{\prime} \sigma_{1} \sigma_{2} \tau_{1} \tau_{2}\right)
\end{aligned}
$$

Table 2. Single particle energies for $\mathrm{Ni}^{58}$

\begin{tabular}{lcc}
\hline $\mathrm{Ni}^{58}$ & $\begin{array}{l}\text { Proton energy } \\
{[\mathrm{MeV}]}\end{array}$ & $\begin{array}{l}\text { Neutron energy } \\
{[\mathrm{MeV}]}\end{array}$ \\
\hline $2 f_{7 / 2}$ & 9.435 & 5.169 \\
$1 h_{11 / 2}$ & 9.088 & 4.233 \\
$1 g_{7 / 2}$ & 7.512 & 2.699 \\
$2 d_{3 / 2}$ & 5.803 & 1.481 \\
$3 s_{1 / 2}$ & 4.848 & -0.080 \\
$2 d_{5 / 2}$ & 4.282 & -0.983 \\
$1 g_{9 / 2}$ & 0.301 & -5.738 \\
$1 f_{5 / 2}$ & -2.497 & -8.659 \\
$2 p_{1 / 2}$ & -2.917 & -8.189 \\
$2 p_{3 / 2}$ & -3.417 & -10.601 \\
$1 f_{7 / 2}$ & -8.177 & -15.498 \\
$2 s_{1 / 2}$ & -12.192 & -20.486 \\
$1 d_{3 / 2}$ & -12.553 & -21.171 \\
$1 d_{5 / 2}$ & -16.058 & -24.593 \\
$1 p_{1 / 2}$ & -21.669 & -31.586 \\
$1 p_{3 / 2}$ & -23.261 & -33.052 \\
\hline
\end{tabular}


Table 3. Parameters of the residual interaction in $\mathrm{Ni}^{58}$

\begin{tabular}{lcccccc}
\hline$V_{0}$ & $F_{p}^{\text {in }}$ & $F_{p}^{\text {ex }}$ & $F_{n}^{\text {in }}$ & $F_{n}^{\text {ex }}$ & $G_{p}$ & $G_{n}$ \\
{$\left[\mathrm{MeV} \mathrm{fm}^{3}\right]$} & & & & & & \\
\hline 380 & 0.65 & -1.39 & -0.85 & -2.29 & 1.3 & -0.3 \\
\hline
\end{tabular}

Table 4. Energy, width and reduced transition probability of the $0^{+}$ and $2^{+}$isoscalar and isovector modes in $\mathrm{Ni}^{58}$

\begin{tabular}{llllc}
\hline$L^{\pi}$ & $T$ & $\begin{array}{l}\hbar \omega \\
{[\mathrm{MeV}]}\end{array}$ & $\begin{array}{l}\Gamma \\
{[\mathrm{MeV}]}\end{array}$ & $\begin{array}{l}B(E L) \uparrow \\
{\left[e^{2} \mathrm{fm}^{4}\right]}\end{array}$ \\
\hline $0^{+}$ & 0 & 17.0 & 0.72 & 932 \\
& 0 & 18.0 & 0.32 & 49 \\
& 1 & 22.0 & 1.00 & 50 \\
& 1 & 28.3 & 4.70 & 220 \\
$2^{+}$ & 0 & 14.6 & 3.19 & 1067 \\
& 0 & 14.9 & 0.30 & 81 \\
& 1 & 24.5 & 4.28 & 121 \\
& 1 & 26.2 & 0.87 & 51 \\
\hline
\end{tabular}

where the parameters $V_{0}, F_{0}, F_{0}^{\prime}, G_{0}$ and $G_{0}^{\prime}$ were fitted to the experimental energies and transition probabilities of the lowest $2^{+}$and $3^{+}$modes and the $1^{-}$giant resonance. As the Migdal force should be universal we took the parameters which are different inside and outside the nucleus close to the $\mathrm{Pb}^{208}$ values [4]. These are shown in Table 3 , defined as in Ref. 3.

The resulting excitation energies of the isoscalar $0^{+}$ and $2^{+}$modes can be approximated by $65 A^{-1 / 3} \mathrm{MeV}$ and $56 A^{-1 / 3} \mathrm{MeV}$ respectively, which is consistent with calculations on other nuclei [4].

The width of these collective modes results mainly from their coupling to single particle continuum states. It can be expressed by [5]

$\Gamma=\frac{2 \pi}{\Delta E} \sum_{i}\left|\left(\hbar \omega-\varepsilon_{i}\right) X_{i}\right|^{2}$

where the sum runs over all continuum states of the chosen configuration space; $\varepsilon_{i}$ is the single particle energy and $\Delta E$ the energy range of continuum states around the energy $\hbar \omega$ of the collective mode. $X_{i}$ is the RPA amplitude.

Table 4 shows energy, width and reduced transition probability of the collective vibrations.

The collectivity of the excitations is expressed by the fraction of the corresponding sum rules which these modes exhaust. The isoscalar monopole and quadrupole sum rules are given by [6]

$\sum_{f} \hbar \omega_{f} \bar{B}(E L)_{f} \uparrow=\frac{(2 L+1)^{2} \hbar^{2} A R^{2}}{(L+2) \pi m} ; \quad L=0,2$.
The sum runs over all final states that can be excited by the multipole operator; $A$ is the mass number, $R$ the $r m s$ radius of the charge distribution of the nucleus and

$\bar{B}(E L)_{f} \uparrow=\left|\left\langle L_{f}^{+}\left\|\sum_{i=1}^{A} r_{i}^{2} Y_{L}^{(i)}\right\| 0^{+}\right\rangle\right|^{2}$

is a modified reduced transition probability, the sum including neutron states.

Summing over all isoscalar excitations with energy up to $24 \mathrm{MeV}$, one finds that most of the sum rule is exhausted: $75.0 \%$ for $L=0$ and $90.5 \%$ for $L=2$.

The scattering experiments [2] show a peak which extends from 13 to $18 \mathrm{MeV}$. The calculated energies and widths of both multipole resonances are thus compatible with the experimental data.

Satchler [2] carried out a DWBA calculation of the differential cross section for the excitation of the monopole as well as the quadrupole mode. For the normalization of the curves he assumed the maximum transition strength allowed by the sum rules.

The differential cross section was measured in the energy region $14.6-16.7 \mathrm{MeV}$. Including the $0^{+}$mode at $17.0 \mathrm{MeV}$ we find in this region only 49.6 and $36.8 \%$ of the $0^{+}$and $2^{+}$sum rules respectively. As the $2^{+}$mode at $14.6 \mathrm{MeV}$ is lying at the edge of the considered region and has a large width we took only

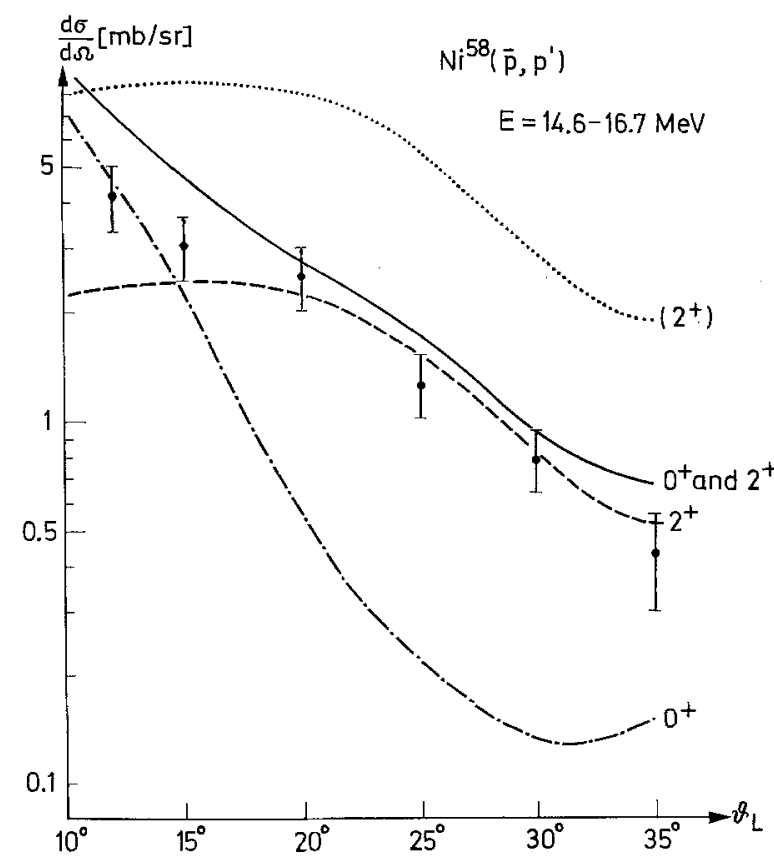

Fig. 1. Cross section for the giant $0^{+}$and $2^{+}$resonances; the data as well as the relative values of the theoretical predictions are taken from Ref. 2 . The dotted DWBA curve labelled with $\left(2^{+}\right)$is normalized as done in Ref. 2 and is shown for comparison. The full line is a superposition of the $0^{+}$and $2^{+}$curves with the proper normalization 
half of its strength when computing the $2^{+}$sum rule fraction.

Fig. 1 shows the renormalized DWBA curves compared with the experimental cross section. The $2^{+}$ curve is in good agreement with the experiment for larger angles while the behavior at small angles is better described by the $0^{+}$mode. The full line denotes the superposition of the $0^{+}$and $2^{+}$cross sections and is in fair agreement with the experimental data in the total angular range considered. If we take the energy region from $12.7-23.7 \mathrm{MeV}$ we find a similar result. Thus is strong evidence that the isoscalar giant resonance excited in proton scattering experiments is a superposition of a monopole and a quadrupole mode.

I wish to thank Dr. P. Ring and R. Bauer for allowing me to use their numerical programs, and Prof. H. Schmidt and Dr. M. Kleber for stimulating discussions.

\section{References}

1. Nagao, M., Torizuka, Y.: Phys. Rev. Lett. 30, 1068 (1973) Lewis, M.B., Bertrand, F.E., Horen, D.J.: Phys. Rev. C8, 398 (1973)

2. Kocher, D.C., Bertrand, F.E., Gross, E.E., Lord, R.S., Newman, E.: Phys. Rev. Lett. 31, 1070 (1973)

3. Ring, P., Bauer, R., Speth, J.: Nucl. Phys. A 206, 97 (1973)

4. Jakubaßa, D. H.: Z. Physik 261, 305 (1973)

5. Fließbach, T.: Z. Physik 218, 385 (1969)

6. Lane, A. M.: Nuclear Theory, p. 79. New York: W.A. Benjamin 1964

Dipl.-Phys. Doris H. Jakubaßa

Physik-Department

Technische Universität München

Theoretisches Teilinstitut

D-8046 Garching, Reaktorgelände

Federal Republic of Germany 